\title{
Discussions on Necessary Conditions for Equivalency of Primary, Irreducible, and Strongly Irreducible Submodules
}

\author{
Najmeh Ahang, Ahmad Khaksari, and Maryam Zandi
}

\begin{abstract}
Among the important types of submodules of a module are maximal submodules, prime and primary submodules. New concepts such as, strongly and strongly irreducible submodules were introduced in last decade. Under some conditions, equality holds for these concepts. Only few conditions that satisfy the equivalency of these types of submodules are known while most of them are yet to be investigated. Some conditions were investigated following the introduction of the concepts of irreducibility and strongly irreducibility of submodules. In this present paper, first the importance of the set of zero divisors was addressed. Then, to create new condition for equivalency, finitely generated modules and multiplicative modules also are applied. Finally, it is shown that the combination of multiplicatively, being finitely generated, and faithfulness of a module is the condition for equivalency
\end{abstract}

Index Terms-Irreducible, Strongly Irreducible, Distributive and Arithmetical Modules.

\section{INTRODUCTION}

In last three decades, considerable researches has been done on rings with distributive lattice of ideals. For example, at 1988 , some properties of rings and distributive lattice of ideals and its generalization on modules was studied by Erdogdu [1] and some properties of distributive modules investigated. A R-module $\mathrm{M}$ is introduced to be distributive if the condition $X \cap(Y+Z)=(X \cap Y)+(X \cap Z)$ for all submodules $X, Y, Z$ of $M$ is satisfied. At 2002, the concept of strongly irreducible ideals was introduced for the first time [2] An ideal $I$ of a ring $R$ is defined Strongly Irreducible (SI) if for ideals $\mathrm{J}$ and $K$ of $R$ the inclusion $J \cap K \subseteq I$ implies that either $J \subseteq I$ or $K \subseteq I$. The concept was generalized at 2006 by Khaksari [3]. In the present paper, [4], the relationship between the irreducible, strongly irreducible, prime, and primary submodules of an R-modules is investigated. Also, one of the most important families of modules which are Laskerian modules is studied. Therefore, some important results on well-known Noetherian modules which are special cases of Laskerian modules are presented. Under some certain conditions, maximal submodules, prime submodules, primary submodules, and SI submodules of a Laskerian module are shown to be equivalent. In section II, we will define some concepts and propositions on zero devisors and its properties which will be used in our proofs in

Manuscript received May 16, 2012; revised June 15, 2012.

N. Ahang and A. Khaksari are with Shiraz Payam-e-Noor University (tel.: +98-9171023389; e-mail: najmeh.ahang@gmail.com).

M. Zandi is with Yazd University, Islamic Republic of Iran. the rest of the paper. Then in section III, the concept of SI will be extended for submodules. Then, using the properties of zero divisors and Nagata's lemma, we will provide the necessary preliminaries for proofs on equivalency on primary, irreducible and strongly irreducible submodules under specific conditions. Then, section IV we discuss the necessary conditions under which these submodules will be equivalent. Lastly, in section $\mathrm{V}$, an specific type of modules will be studied where four types of submodules - which are prime, primary, irreducible, and strongly irreducible submodules are equivalent.

\section{INTRODUCTION TO SOME CONCEPTS OF COMMUTATATIVE} ALGEBRA

Definition: Let $P$ be an ideal of $R$ and $P \neq R$, then for each ideal $A, B$ in $R$ :

$$
A B \subseteq P \Rightarrow(B \subseteq P) \operatorname{or}(A \subseteq P)
$$

Definition: Let $Q$ be the ideal of R. $Q$ is primary ideal if for each $x, Y \in R$ :

$$
x y \in R \Rightarrow y \in \operatorname{Ror} \exists n \in I N \text { э } x^{n} \in Q
$$

Definition: Let $\mathrm{R}$ be a commutative and unitary ring and $I$, $J, K$ be ideals of $R$. Then $I$ is irreducible if:

$$
I=K \bigcap J \Rightarrow I=K o r I=J
$$

Definition: Let $R$ be a commutative and unitary ring and $I$, $J, K$ be ideals of $R$. Then $I$ is strongly irreducible ideal if:

$$
J \cap K \subseteq I \Rightarrow K \subseteq I \text { IorJ } \subseteq I
$$

Definition: Let $S$ be a subset of commutative ring $R$. $S$ is closed multiplicative subset if:

1) $0 \notin S$

2) $1 \in S$

3) if $s_{1}, s_{2} \in S \Rightarrow s_{1} s_{2} \in S$

Definition: Let $R$ be a commutative and unitary ring, $M$ be a R-module and $S$ be a closed multiplicative subset of $R$. Then:

$$
\left(m_{1}, s_{1}\right) \sim\left(m_{2}, s_{2}\right) \Leftrightarrow \exists l \in S, l\left(m_{1} s_{2}-s_{1} m_{2}\right)=0
$$

This multiplication is an equivalence relation on $M \times S$. In this paper $[(\mathrm{m}, \mathrm{s})]$ is denoted as $\frac{m}{s}$ and the set of all equivalence classes as follows: 


$$
S^{-1} M=\left\{\frac{m}{s} \mid m \in M, s \in S\right\}
$$

Remark: If $P$ be a prime ideal of $R, S=R-P$ then $S$ is a closed multiplicative subset of R. $S^{-1} M$ is denoted as $M_{p}$.

Definition: Let $M$ be a ring and $I, J, K$ ideals of $R . R$ is arithmetical if:

$$
\begin{aligned}
(I+J) \cap K & =(I \cap K)+(J \cap K) \operatorname{or}(I \cap J)+K \\
& =(I+K) \cap(J+K)
\end{aligned}
$$

Definition: Let $M$ be a $R$-module and $K, P, N$ submodules of $M . M$ is distributive if:

$$
N \cap(P+K)=(N \cap P)+(N \cap K)
$$

Definition: Let $I$ be an ideal of $R$. The following definition exists:

$$
r(I)=\sqrt{I}=\left\{x \in R \mid \exists n \in I N \ni x^{n} \in I\right\}
$$

Preposition: Let $I$ be an ideal of $R$. Then it can be proved that $r(I)=\bigcap_{p \in \operatorname{spec}(R), I \subseteq P} P,[4]$

Definition: Let $P, N$ be $R$-modules. Then $(N: P)=\{x \in R \mid x P \subseteq N\}$ If $N=0$ then $(0: P)=$

$$
Z_{R}\left(\frac{R}{(N: M)}\right)=\{r \in R \mid \exists x \in R \ni r x \in(N: M), x \notin(N: M)=\{r \in R \mid \exists x \in R \ni r x M, \subseteq N, x \notin(N: M)\}
$$

Using equation (11), it can be shown that there exists $s \in R$ such that $s \neq 0$ and $\mathrm{s} \notin(N: M)$ and $r s \in(N: M)$. Also

$$
\begin{aligned}
Z_{R}\left(\frac{M}{N}\right) & =\left\{r \in R \mid \exists n \in \frac{M}{N} \ni r(n+N)=N\right\} \\
& =\left\{r \in R \mid \exists n \in \frac{M}{N} \ni r n \in N\right\}
\end{aligned}
$$

Since $s \notin(\mathrm{N}: \mathrm{M})$, then there exist $m \in \mathrm{M}$ such that sm $\notin$ $N$ and $r s \in(N: M)$. Hence, $r s M \subseteq N$ and rsm $\in N$. So $s m$ is the member that satisfies the definition of $Z_{R}\left(\frac{M}{N}\right)$ and therefore $r \in Z_{R}\left(\frac{M}{N}\right)$

Lemma: Let $\mathrm{M}$ be a R-module and $\mathrm{N}$ be a submodule of $\mathrm{M}$. Then $Z_{R}\left(\frac{M}{N}\right)$ is a prime ideal of $\mathrm{R}$ iff $Z_{R\left(\frac{R}{N: M}\right)}\left(\frac{M}{N}\right)$ be a prime ideal of $\left(\frac{R}{N: M}\right)[3]$.

\section{Strongly IRREDUCIBLE (SI) SUBMOdULES}

Definition: Let $M$ be a R-module and $N, K, L$ be submodules of $M$. Then, $\mathrm{N}$ is strongly irreducible submodule if:
$A_{n n R}(P)$.

Definition: The $R$-module $M$ is faithful if $A_{n n R}(M)=0$.

Lemma: If $M$ be a R-module, $I$ be an ideal of $R$ and $A_{n n R}(M)$ $=I$, then $M$ as a $\frac{R}{I}$ module is faithful.

Proof: Immediate from the last definition

Definition: Let $M$ be a $R$-module. Then, $Z_{R}(M)=\{r \in R \mid \exists m \in M$ э $m \neq 0, r m=0\}$

Lemma: Let $M$ be a $R$-module and $S=\left\{r \in R \mid r \notin Z_{R}(M)\right\}$, then it can be proved that $S$ is a closed multiplicative subset of $R$, [4].

Proposition: Let $M$ be a $R$-module. Then $Z_{R}(M)$ is the union of some prime ideals of $R$, [4].

Lemma: Let $\mathrm{M}$ be a R-module and $\mathrm{N}$ be a submodule of $M$. Then:

$$
Z_{R}\left(\frac{R}{(N: M)}\right) \subseteq Z_{R}\left(\frac{M}{N}\right)
$$

Proof: Let $r \in Z_{R}\left(\frac{R}{(N: M)}\right)$ Then:

$$
L \bigcap K \subseteq N \Rightarrow K \subseteq N o r L \subseteq N
$$

Definition: Let $N$ be a primary submodule of $R$-module $\mathrm{M}$ and $P=r(N: M)$. Then, $N$ is $P$-primary submodule of $R$-module $M$.

Proposition: Let $N$ be a submodule of $R$-module $M$. Then, $Z_{R}\left(\frac{M}{N}\right)$ is prime ideal of $R$.

Proof: Firstly it should be proved that $Z_{R}\left(\frac{M}{N}\right)$ is ideal. For this purpose, let $r_{1}, r_{2} \in Z_{R}\left(\frac{M}{N}\right)$.

According to definition :

$$
\exists m_{1}, m_{2} \in\left(\frac{M}{N}\right) \ni r_{1}\left(m_{1}+N\right)=N, r_{2}\left(m_{2}+N\right)=N
$$

Since $\mathrm{M}$ is strongly irreducible, then $R m_{1} \cap R m_{2} \not \subset N$ If $R m_{1} \cap R m_{2} \subset N$ then $N$ being $S I$ gives:

$R m_{1} \subseteq N$ or $R m_{2} \subseteq N$ and since $R$ is unitary then, $m_{1} \in N$ or $m_{2} \in N$ and this is a contradiction.

Now, let $x \in R m_{1} \cap R m_{2} \subseteq N$. Then, there exist $\mathrm{a}_{1}, \mathrm{a}_{2}$ $\in R$ so that $x=a_{1} m_{1}=a_{2} m_{2}$. Since $r_{1} m_{1} \in N$, then $r_{1} x=$ $\mathrm{a}_{1} \mathrm{r}_{1} \mathrm{~m}_{1} \in \mathrm{N}$ and $\mathrm{r}_{2} \mathrm{x}=\mathrm{a}_{2} \mathrm{r}_{2} \mathrm{~m}_{2} \in \mathrm{N}$.

Hence: 


$$
\left(r_{1}+r_{2}\right) x \in N, r_{1}+r_{2} \in Z_{R}\left(\frac{M}{N}\right)
$$

Also, to prove that $Z_{R}\left(\frac{M}{N}\right)$ is ideal, let $t \in R, r \in$ $Z_{R}\left(\frac{M}{N}\right)$ Using the definition of $Z_{R}\left(\frac{M}{N}\right)$, there exist $n$ $\in\left(\frac{M}{N}\right)$ such that $r(n+N)=N$ which gives $r n \in N$.

Also, $N$ is a submodule of $M$ that results in $\operatorname{trn} \in \mathrm{N}$, $t r$ $\in Z_{R}\left(\frac{M}{N}\right)$. Hence $Z_{R}\left(\frac{M}{N}\right)$ is an ideal of $R$.

To prove that $Z_{R}\left(\frac{M}{N}\right)$ is prime ideal, let:

$$
r_{1} r_{2} \in Z_{R}\left(\frac{M}{N}\right)
$$

Using the definition, there exist $m \in\left(\frac{M}{N}\right)$ such that $r_{1} r_{2}(m+N)=N$. Then, $r_{1} r_{2} m \in N$. Let:

$$
r_{1}, r_{2} \notin Z_{R}\left(\frac{M}{N}\right)
$$

then, for each $m \in \frac{M}{N}$ the following relation holds:

$$
r_{1} m \notin N, r_{2} m \notin N
$$

Also, $r_{1}, r_{2} \in N$ shows that $r_{1}\left(r_{2} m\right) \in N$. Hence, $r_{2}\left(r_{1} m\right) \in N$ and it is proved that $r_{2} \in Z_{R}\left(\frac{M}{N}\right)$ which leads to a contradiction.

Hence

$$
r_{1} \in Z_{R}\left(\frac{M}{N}\right), \text { or }, r_{2} \in Z_{R}\left(\frac{M}{N}\right)
$$

Corollary: Let $N$ be a strongly irreducible submodule of $R$-module $M$ and $I=(N: M)$. Then, $Z_{\frac{R}{I}}\left(\frac{M}{N}\right)$ is prime ideal of $\frac{R}{I}$

Proof: Immediate from above proposition.

Corollary: If I be a (SI) ideal of R, then $Z_{\frac{R}{I}}\left(\frac{R}{I}\right)$ is prime ideal of $\frac{R}{I}$

Definition: Let $M$ be a $R$-module and $N$ be a submodule of $M$. Then,

$$
r(N)=\left\{r \in R \mid \exists n \in I N \text { э } x^{n} M \subseteq N\right\}
$$

Lemma(Nagata's lemma) [6]: Let Q be a P-primary ideal of $\mathrm{R}$ and $\mathrm{S}$ be a closed multiplicative submodule of $\mathrm{R}$. Then:
1) If $P \bigcap S \neq \phi$ then $\mathrm{PRs}=\mathrm{QRs}=$ Rs.

2) If $P \cap S=\phi$ then QRs is PRs -primary ideal and:

$$
P R_{s} \cap R=P, Q R_{s} \cap R=Q
$$

Proposition: Let $I$ be an ideal of arithmetical ring $R$. Then, $I$ is irreducible iff $I$ be (SI) ideal iff the set of all zero divisors of $\frac{R}{I}$ be a prime ideal of $R$.

Proof: The ideal $I$ of $R$ is irreducible if it is not in the form of intersection of two ideal of $R$. If $I$ be irreducible and $K$, $J \quad$ be the ideals of $R$ such that:

$$
(J \cap K) \subseteq I,(J \cap K)+I=(J+I) \cap(K+I)(21)
$$

Then,

$$
K \subseteq I, o r, J \subseteq I
$$

Therefor I is irreducible. According to the definitions of strongly irreducible and irreducible ideals, irreducibility is immediate result of being strongly irreducible. First part of proposition is proved here.

To prove the second part, let the set of zero devisors of $\frac{R}{I}$ be a prime ideal P. Then, Nagata's lemma shows $I=I R_{P}$ $\bigcap R$.

It is concluded that $R_{P}$ is totally ordered set, [3]. Therefore, $I R_{P}$ as a ideal of $R_{P}$ is SI. Hence $I$ is $\mathrm{SI}$.

\section{IV. (SI) Submodules In Multiplication Modules}

Definition: A proper submodule $K$ of a module $M$ over a ring $\mathrm{R}$ is said to be prime (resp primary) if $r a \in K$ for $\mathrm{r} \in \mathrm{R}$ and $a \in M / K$ implies that $r M \subseteq K\left(\operatorname{resp} r^{n} M \subseteq K\right.$ for some $n$ $\subseteq N$ ).

Proposition 1: Let $M$ be a multiplication and $N$ be a prime submodule of $M$. Then, $N$ is a SI submodule, [3].

Remark: Each commutative ring with identity is cyclic. Therefore, each of its SI ideal is strongly irreducible submodule of cyclic multiplication R-module.

Corollary: Let $R$ be a commutative and unitary ring. Then, each ideal of $R$ is SI.

Proposition2: Let $M$ be a finitely generated multiplication faithful module and $N=I M$ be a submodule. Then,

1) Let $I_{l}$ be an ideal of $R$, then $I_{l} M \subseteq I M$ iff $I_{l} \subseteq I$.

2) $N=I M$ is a prime sub-module iff $I$ is a prime ideal of $R$.

3) $N=I M$ is SI iff I is SI.

4) If $P$ be a prime ideal of $R$, then $P M$ is a SI submodule.

5) $I$ is a primary ideal of $R$ iff $N=I M$ is primary submodule.

Proof: Refer to [4].

Proposition 3: Let $M$ be a multiplication $R$-module and $P$ be a prime ideal of $R$. Then, there exist a SI submodule $N$ of $M$ so that $Z_{R}(M / N)=P$.

Proof: The proof is immediate from proposition 2.

Proposition 4: A multiplication faithful $R$-module $M$ on $R$-domain is finitely generated [5].

Proposition 5: Let $M$ be a distributive R-module. Then, each primary submodule is SI submodule [1]. 
Proposition 6: Let $R$ be a PID and $M$ be a multiplication faithful R-module. Then, each $P$-primary submodule $N$ of $M$ is strongly irreducible, [4].

Corollary: Let $R$ be a Dedekind domain and $M$ be a faithful multiplication module and $\mathrm{N}$ be a submodule of $M$. Then, the following statements are equivalent:

1) $N$ is primary.

2) $\mathrm{N}$ is $\mathrm{SI}$.

3) $\mathrm{N}$ is irreducible.

Proposition 7: Let $M$ be a distributive $R$-module and $\mathrm{N}$ be a submodule. Then, $N$ is strongly irreducible iff $N$ be irreducible, [4].

Proposition 8: Let $R$ be an arithmetical ring and $N$ be a finitely multiplicative $R$-module and $N$ be a submodule of $M$. Then, $Z_{R}(\mathrm{M} / \mathrm{N})$ is prime ideal iff $N$ is strongly irreducible iff $N$ is irreducible. Also, if $N$ is a primary submodule, then $N$ is irreducible.

Proof: To prove above mentioned proposition, the following statements have to be established step by step:

1) If $\mathrm{N}$ be a SI submodule, then $Z_{R}\left(\frac{M}{N}\right)$ is a prime ideal of $R,[5]$.

2) If $Z_{R}\left(\frac{M}{N}\right)$ be a prime ideal of $\mathrm{R}$, then $N$ is a $\mathrm{SI}$ submodule of $M$. To prove this statement, let $N$ be a submodule of $M$ and $I$ be an ideal of $\mathrm{R}$ so that $N=I M$ and $Z_{R}\left(\frac{M}{N}\right)$ be an prime ideal of $\mathrm{R}$. Define a module of quotient $\left(\frac{M}{N}\right)$ Since $M$ is multiplicative and finitely generated, then $\left(\frac{M}{N}\right)$ as a $\frac{R}{I}$ module is multiplicative, finitely generated and faithful.

So according to proposition 2 , that is enough to prove $I$ is SI.

Last proposition of section II proves that if $R$ be an arithmetical ring and $I$ be an its ideal, then $Z_{R}\left(\frac{R}{I}\right)$ is prime ideal iff I be SI. Also

$$
Z_{R}\left(\frac{M}{N}\right)=Z_{\frac{R}{I}}\left(\frac{R}{I}\right)
$$

Since $Z_{R}\left(\frac{M}{N}\right)$ is prime, then $Z_{\frac{R}{I}}\left(\frac{M}{N}\right)$ is prime. Hence, $Z_{R}\left(\frac{R}{I}\right)$ is prime which gives I is $\mathrm{SI}$ and as the result $\mathrm{N}$ is $\mathrm{SI}$.

3) If $N$ be a SI submodule of $M$, then $N$ is irreducible.

4) If $R$ be an arithmetical ring and $M$ be a multiplicative and finitely generated module and $N$ be an irreducible submodule of $M$, then $N$ is SI.

5) If $R$ be an arithmetical and finitely generated ring then, $M$ is multiplicative iff is a distributive and according to proposition7, if $\mathrm{M}$ be a distributive module, then $N$ is SI iff is irreducible submodule of $M$. So, according to this results $N$ is SI.

6) If $\mathrm{N}$ be a primary submodule of $M$, then $\mathrm{N}$ is irreducible submodule of $M$. To prove this statement let $\mathrm{N}$ be a primary submodule of $M$ and for arbitrary ideal $\mathrm{I}$ of $\mathrm{R}$ there exist submodule $\mathrm{N}$ of $M$ so that $N=I M$.

Also, let be submodules of M so that $N_{1} \bigcap N_{2}=N$. Since $M$ is multiplicative module, then there exist ideals $I_{1}, I_{2}$ so that:

$$
N_{1}=I_{1} M, N_{2}=I_{2} M
$$

Then, $N=\left(\mathrm{I}_{1} \bigcap \mathrm{I}_{2}\right) \mathrm{M}$. Also, $N$ is a primary submodule. Then, $I$ is a primary ideal of $R$ so I is SI, [7]. Hence $I=I_{1}$ or $I$ $=I_{2}$ then, $N=N_{1}$ or $N=N_{2}$.

Proposition 9: Let $\mathrm{N}$ be a submodule of multiplicative $R$-module $N$. Then, $N=(\operatorname{Ann}(M / N)) M=(N: M) M$.

Proof: Let $A=A n n\left(\frac{M}{N}\right)$ Since $M$ is a multiplicative module, then there exist an ideal I of $R$ such that $N=I M$.

Therefore:

$$
I \subseteq A, N=I M \subseteq A M \subseteq N
$$

Hence, $N=A M$.

Proposition 10: Let $M$ be a multiplicative p R-module and $N=I M$ be a SI submodule. Then, $N$ is a prime submodule iff $\sqrt{(N: M)}=(N: M)$

Lemma: Let $N$ be a primary submodule of $M$ and $P=r(N$ : $M)$ and $\mathrm{S}$ be a closed multiplicative subset of $\mathrm{R}$ such that $P \bigcap S=\phi$. Then, $N=N_{P} \cap M$

Since $N \bigcap M$ and $N \cap N P$, then $N \subseteq N P \bigcap M$. Using proof by contradiction, we prove that $N P \bigcap M \subseteq N$.

Let, $x=\frac{n}{s} \in N_{P} \bigcap M$ such that $x \notin N$ by the definition of $N P$, there exist $t \in S$ such that stx $\in N$ and hence $s t \in P$ and it is contradiction.

Proof: $(\Rightarrow)$ If $\mathrm{N}$ be a prime submodule of $\mathrm{M}$, then it can be shown from $N=(N: M) M$ and proposition 2 that $(N: M)$ is prime ideal of $R$. Therefore, using the above mentioned lemma we have:

$$
\sqrt{(N: M)}=(N: M)
$$

$(\Leftarrow)$ Let $\mathrm{N}$ be a SI submodule of $\mathrm{M}$ and $\sqrt{(N: M)}=(N: M)$, it is sufficient to prove that $(N: M)$ is prime ideal.

Therefore, let $A, B$ be ideals of $\mathrm{R}$ such that $A B \subseteq(N: M)$. Hence:

$$
A \cap B \subseteq \sqrt{A \cap B} \subseteq \sqrt{A B} \subseteq \sqrt{(N: M)}=(N: M)
$$

Since $(N: M)$ is SI ideal of $R$, then $A \subseteq(N: M)$ or $B \subseteq(N$ : $M)$. Hence $(\mathrm{N}: \mathrm{M})$ is prime ideal and therefore $N=(N: M) M$ is a prime submodule of $M$.

Lemma: Let $M$ be a faithful finitely generated multiplicative $R$-module and $N=(N: M) M$ be a sub-module of $M$. Then, there exists a SI minimal submodule of $\mathrm{M}$ so that it includes $M$, [4]. 
Proposition 11: Let $\mathrm{R}$ be an UFD and $\mathrm{I}$ be an ideal of $\mathrm{R}$ and $x, y \in R$, then $I$ is a SI ideal iff $[x, y] \in I$ which implies $x$ $\in I$ or $y \in I$ - where $[x, y]$ represents Least Common Multiple (LCM) of $x$ and $y$ - [3].

Corollary: Let $R$ be an UFD and $M$ be a faithful multiplication $R$-module. Then, $\mathrm{N}=\mathrm{IM}$ is a SI submodule iff $x, y \in R,[x, y] \in I) \Rightarrow(x \in I)$ or $(y \in I)$.

Proposition 12: Let $R$ be an UFD and $M$ a faithful multiplication $R$-module and $a \in R$. Then, $I N=\langle 2\rangle M$ is a SI submodule iff there is $p \in R$ (p is prime devisor) and $n$ $\in I N$ so that $I N=\left\langle p^{n}\right\rangle M$

Proposition 13: Let $\mathrm{R}$ be an UFD and $M$ be a multiplication faithful R-module and $N=I M$ be SI submodule of $M$. Then, $N$ is a primary submodule of $M$, [3].

Proposition 14: Let $\mathrm{R}$ be an UFD and $M$ be a multiplication faithful module and $N=\langle a\rangle M$ be a submodule of $M$. Then, $N$ is SI iff $N$ is primary, [3].

\section{TOTALLY FLAT RINGS AND LASKERIAN MODULES}

Definition: A commutative ring $R$ with identity is totally flat if each of its principle ideals is an idempotent ideal.

Definition: Let $M$ be a R-module. Then $M$ is called Laskerian module if each sub-module of $M$ is a finite intersection of primary submodules of $M$.

Remark: Good example for Laskerian modules are Noetherian modules.

Proposition: Let $R$ be a totally flat ring and $M$ be a faithful multiplication and finitely generated module and $N=I M$ be a submodule of $M$. Then, the following statements are equivalent:

1) $N$ is a maximal submodule of $M$.

2) $N$ is a prime submodule of $M$.

3) $N$ is a primary submodule of $M$.

4) $N$ is a strongly irreducible submodule of $M$.

Proposition: Let $R$ be a Laskerian ring and $M$ be a faithful multiplication and finitely generated module and $N=I M$ be a submodule of $M$. So, if $\mathrm{N}$ is SI submodule, then $N$ is primary submodule of $M$.

Corollary: Let R be a Noetherian ring and $M$ be a faithful multiplication and finitely generated module. Then, each irreducible submodule of $M$ is a primary submodule of $M$.

\section{REFERENCES}

[1] V. Erdogdu, "Multiplication modules which are distributive," Journal of Pure and Applied Algebra, vol. 54, issues 2-3, pp. 209-213, October 1988.

[2] W. J. Heinzer, L. J. Ratliff, and D. E. Rush, "Strongly irreducible ideals of a commutative ring," Journal of Pure and Applied Algebra, vol. 166, pp. 267-275, 2002.

[3] A. Khaksari, M. Ershad, and H. Sharif, "Strongly Irreducible Submodules of Modules," Acta Mathematica Sinica, vol. 22, issue 4, pp. 1189-1196, 2006.

[4] N. Ahang, "Some Results on Strongly irreducible Submodules," Payam-e-Noor University Publications, Shiraz, Feb 2008.

[5] Z. A. El-Bast and P. F. Smith, "Multiplication modules," Comm. Algebra, vol. 16, pp. 755-779, 1988.

[6] C. Musili, Introduction to Rings and modules, Narosa Pub House, 2 edition, 1997.

[7] C. Jensen, “Arithmetical Rings," Acta Math. Sci Hungar, vol. 17, pp. 115-123, 1966. 